\title{
PRODUCTS OF $k$-SPACES AND SPACES OF COUNTABLE TIGHTNESS
}

BY

\author{
G. GRUENHAGE AND Y. TANAKA
}

\begin{abstract}
In this paper, we obtain results of the following type: if $f: X \rightarrow Y$ is a closed map and $X$ is some "nice" space, and $Y^{2}$ is a $k$-space or has countable tightness, then the boundary of the inverse image of each point of $Y$ is "small" in some sense, e.g., Lindelöf or $\omega_{1}$-compact. We then apply these results to more special cases. Most of these applications combine the "smallness" of the boundaries of the point-inverses obtained from the earlier results with "nice" properties of the domain to yield "nice" properties on the range.
\end{abstract}

Introduction. Recall the following theorem due to Morita and Hanai [14] and Stone [17].

Theorem. If $f: X \rightarrow Y$ is closed and $X$ is metrizable, then the following are equivalent.

(a) $Y$ is first countable;

(b) For each $y \in Y, \partial f^{-1}(y)$ is compact;

(c) $Y$ is metrizable.

The (c) $\Rightarrow$ (b) part is due to Vaĭnsteĭn [22]. But even the (a) $\Rightarrow$ (b) part holds under much more general conditions: Michael [7] showed (b) holds if $X$ is paracompact, and $Y$ is locally compact or first-countable.

Note that the assumptions on $Y$ in Michael's theorem could not be weakened to " $Y$ is a $k$-space" or " $Y$ has countable tightness": the map identifying the limit points of a topological sum of $\kappa$ convergent sequences is a closed map from a metrizable space $X$ to a Fréchet space $Y$, and $\left|\partial f^{-1}(y)\right|=\kappa$ for some $y \in Y$. In this paper, we show that the situation is different if we require $Y^{2}$ to be a $k$-space or have countable tightness. (Recall that the square of a $k$-space or a space of countable tightness need not have the same property.) We will usually not be able to show that the boundaries of point-inverses are compact, but we will often (depending upon conditions imposed on $X$ or $Y$ ) be able to show that they are "small" in some sense, e.g., Lindelöf or $\omega_{1}$-compact. In the second section, we apply general results of this type to more special cases, often combining the "smallness" of the boundaries of point-inverses with "nice" properties of $X$ to obtain "nice" properties of $Y$.

We mention the following earlier result of the second author [21] which is related to this topic.

Received by the editors February 11, 1980 and, in revised form, July 13, 1981.

1980 Mathematics Subject Classification. Primary 54D50; Secondary 54A25, 54C10, 54D55. 
Theorem. If $f: X \rightarrow Y$ is closed and $X$ is metrizable, then the following are equivalent.

(a) For each $y \in Y, \partial f^{-1}(y)$ is Lindelöf.

(b) Y has a point-countable k-network [see §2, Definition 2.1].

(c) $Y$ has a $\sigma$-locally-countable $k$-network.

See [7] and [21] for other related results.

We will often make use of the following well-known property of closed maps (cf. [3,p. 52]): If $f: X \rightarrow Y$ is closed, then for each $y \in Y$ and open $U \subset X$ such that $f^{-1}(y) \subset U$, there is a neighborhood $V$ of $y$ such that $f^{-1}(V) \subset U$.

1. General results. All our spaces are assumed to be regular and $T_{1}$. We consider cardinals to be initial ordinals. We now recall some basic definitions.

Definition 1.1. A space $X$ has the weak topology with respect to a collection $C$ of sets if a subset $A$ of $X$ is closed (resp., open) in $X$ if and only if $A \cap C$ is closed (resp., open) in $C$ for each $C \in \mathcal{C}$.

Definition 1.2. A space $X$ is a $k$-space (quasi-k-space) if $X$ has the weak topology with respect to its compact (countably compact) subsets. $X$ is sequential if $X$ has the weak topology with respect to its compact metric subspaces (equivalently, with respect to its subspaces homeomorphic to $\omega+1$, a sequence with its limit point). $X$ has countable tightness (denoted by $t(X) \leqslant \omega$ ) if it has the weak topology with respect to its countable subsets.

We will be using the following elementary facts about these concepts.

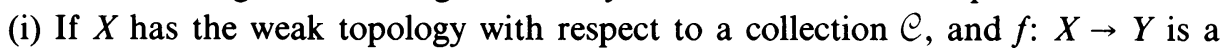
quotient map, then $Y$ has the weak topology with respect to $\{f(C): C \in \mathcal{C}\}$. Thus all properties named in Definition 1.2 are preserved by quotient maps.

(ii) If $X$ satisfies any of the properties in Definition 2.2 locally, then the whole space has the property.

(iii) If $X$ has a locally finite cover by a family $\mathcal{C}$ of closed sets, then $X$ has the weak topology with respect to $C$.

Definition 1.3. A space $X$ is (strongly) collectionwise Hausdorff if whenever $\left\{x_{\alpha}\right.$ : $\alpha \in A\}$ is a closed discrete subset of $X$, there exists a (discrete) disjoint collection $\left\{U_{\alpha}: \alpha \in A\right\}$ of open sets such that $x_{\alpha} \in U_{\alpha}$ for each $\alpha \in A$.

Note that every normal collectionwise Hausdorff space is strongly collectionwise Hausdorff.

Let $c$ denote the cardinality of the continuum.

THEOREM 1.4. Suppose $f: X \rightarrow Y$ is closed, with $X$ strongly collectionwise Hausdorff. Then the boundary, $\partial f^{-1}(y)$, of $f^{-1}(y)$ is c-compact for each $y \in Y$ if either

(a) $Y^{2}$ is quasi-k and $t(Y) \leqslant \omega$ or

(b) $t\left(Y^{2}\right) \leqslant \omega$.

Proof. Suppose $\partial f^{-1}(y)$ is not $c$-compact. Then there is a closed discrete subset $D \subset \partial f^{-1}(y)$, with $|D|=c$. For each $d \in D$, let $U_{d}^{\prime}$ be an open set containing $d$ such that $\left\{U_{d}^{\prime}: d \in D\right\}$ is discrete.

Let $d \in U_{d} \subset \bar{U}_{d} \subset U_{d}^{\prime}$, where $U_{d}$ is open. Note that $\left\{U_{d}: d \in D\right\}$ is also discrete. 
For each $d \in D, y \in \overline{f\left(U_{d}-f^{-1}(y)\right)}$. Since $t(Y) \leqslant \omega$, there is a countable set $\left\{y_{d, n}: n \in \omega\right\} \subset f\left(U_{d}-f^{-1}(y)\right)$ such that $y \in\left\{\overline{y_{d, n}: n \in \omega}\right\}$.

Let $X_{d, n}=f^{-1}\left(y_{d, n}\right) \cap \bar{U}_{d}$, and let $X_{d}=f^{-1}(y) \cap \bar{U}_{d}$.

If $O$ is open and contains $X_{d}$, then there is an open set $O^{\prime}$ such that $O^{\prime} \cap \bar{U}_{d}=\varnothing$, and $f^{-1}(y) \subset O \cup O^{\prime}$. Let $W$ be the complement in $Y$ of $f\left(X-\left(O \cup O^{\prime}\right)\right)$. Then $y \in W$, so there is $n \in \omega$ such that $y_{d, n} \in W$, and hence $f^{-1}\left(y_{d, n}\right) \subset O \cup O^{\prime}$. Thus $X_{d, n} \subset\left(O \cup O^{\prime}\right) \cap \bar{U}_{d}=O \cap \bar{U}_{d} \subset O$. Choose $x_{d, n} \in X_{d, n}$, and let $A_{d}=\left\{x_{d, n} ; n \in\right.$ $\omega\}$. By the above argument, every open set containing $X_{d}$ contains infinitely many elements of $A_{d}$.

For $x \in A_{d}$, let $D_{x}=\left\{d^{\prime} \in D\right.$ : there exists $x^{\prime} \in \bar{U}_{d^{\prime}}$ with $\left.f(x)=f\left(x^{\prime}\right)\right\}$. Let $B_{d}=\left\{x \in A_{d}: D_{x}\right.$ is uncountable $\}$.

Claim 1. $X_{d} \cap \bar{B}_{d}=\varnothing$. To see this, let $B_{d}=\left\{x_{0}, x_{1}, \ldots\right\}$. Inductively choose a sequence $d_{0}, d_{1}, \ldots$ of distinct elements of $D$, and points $x_{n}^{\prime} \in \bar{U}_{d_{n}}$ such that $f\left(x_{n}^{\prime}\right)=f\left(x_{n}\right)$. Then $\left\{x_{0}^{\prime}, x_{1}^{\prime}, \ldots\right\}$ is a closed subset of $X$, so $f\left(\left\{x_{0}^{\prime}, x_{1}^{\prime}, \ldots\right\}\right)=f\left(B_{d}\right)$ is closed. Thus $y \notin f\left(B_{d}\right)=\overline{f\left(B_{d}\right)}=f\left(\bar{B}_{d}\right)$, so $X_{d} \cap \bar{B}_{d}=\varnothing$.

Let $C_{d}=A_{d}-B_{d}$. By Claim $1, X_{d} \cap \bar{C}_{d} \neq \varnothing$. Pick $d(0) \in D$. Let $D(d(0))=$ $\cup\left\{D_{x} ; x \in \bar{C}_{d(0)}\right\}$. Observe that $D(d(0))$ is countable. If $d(\beta)$ has been chosen for all $\beta<\alpha<c$, let $d(\alpha) \in D-\cup_{\beta<\alpha} D(d(\beta))$. Observe that if $x \in C_{d(\beta)}$ and $x^{\prime} \in$ $C_{d(\alpha)}$ with $\alpha \neq \beta$, then $f(x) \neq f\left(x^{\prime}\right)$.

Now let $\mathcal{E}=\left\{E_{\alpha}: \alpha<c\right\}$ index all subsets of $\cup_{n \in \omega} C_{d(n)}$ such that $\left|E_{\alpha} \cap C_{d(n)}\right|=1$ for each $\alpha<c$ and $n \in \omega$. Let $E_{\alpha}=\left\{e_{\alpha n} ; n \in \omega\right\}$ such that $e_{\alpha n} \in C_{d(n)}$. Let $C_{d(\alpha)}=$ $\left\{c_{\alpha n} ; n \in \omega\right\}$.

For $x \in X$, denote $f(x)$ by $x^{*}$. Let $H_{\alpha}=\left\{\left(e_{\alpha n}^{*}, c_{\lambda_{\alpha} n}^{*}\right): n \in \omega\right\} \subset Y^{2}$, where $\lambda_{\alpha}$ is the $\alpha$ th limit ordinal, and let $H=\cup_{\alpha<c} H_{\alpha}$.

Claim 2. $(y, y) \in \bar{H}-H$. To see this, suppose $y \in O, O$ open in $Y$. We know $X_{d(\alpha)} \cap \bar{C}_{d(\alpha)} \neq \varnothing$, so $y \in f\left(\bar{C}_{d(\alpha)}\right)=\overline{f\left(C_{d(\alpha)}\right)}$. For each $n \in \omega$, choose $x_{n} \in C_{d(n)}$ such that $x_{n}^{*} \in O$. Then $\left\{x_{n}: n \in \omega\right\}=E_{\alpha}$ for some $\alpha$, and $e_{\alpha n}=x_{n}$ for each $n$. There is $n \in \omega$ such that $c_{\lambda_{\alpha} n}^{*} \in O$. Thus $\left(e_{\alpha n}^{*}, c_{\lambda_{\alpha} n}^{*}\right) \in O^{2} \cap H_{\alpha}$, which proves the claim.

The next claim completes the proof of part (a).

Claim 3. If $K \subset Y^{2}$ is countably compact, then $K \cap H$ is finite. To see this, suppose $\alpha_{0}, \alpha_{1} \ldots$ are distinct ordinals such that for each $n \in \omega, K \cap H_{\alpha_{n}} \neq \varnothing$. Then we can find $\left(e_{\alpha_{n} k_{n}}^{*}, c_{\lambda_{\alpha_{n}} k_{n}}^{*}\right) \in K \cap H_{\alpha_{n}}$. But $\left\{c_{\lambda_{\alpha_{n}} k_{n}}: n \in \omega\right\}$ is a closed discrete subset of $X$, since $c_{\lambda_{\alpha_{n}} k_{n}} \in \vec{U}_{d\left(\lambda_{\alpha_{n}}\right)}$. Thus $\left\{\left(e_{\alpha_{n} k_{n}}^{*}, c_{\lambda_{\alpha_{n}} k_{n}}^{*}\right) ; n \in \omega\right\}$ is an infinite closed discrete subset of $K$, contradiction. Thus $K$ meets only finitely many $H_{\alpha}$ 's. Now suppose that for fixed $\alpha, K \cap H_{\alpha}$ is infinite. Then for each $n \in \omega$, we can find $\left(e_{\alpha k_{n}}^{*}, c_{\lambda_{\alpha} k_{n}}^{*}\right) \in K \cap H_{\alpha}$. But $\left\{e_{\alpha k_{n}}: n \in \omega\right\}$ is an infinite closed discrete subset of $X$ and we get a contradiction as before. Thus each $K \cap H_{\alpha}$ is finite, and so $K \cap H$ is finite.

To complete the proof of part (b), we have the next claim.

Claim 4. No countable subset of $H$ contains $(y, y)$ in its closure. Suppose $C \subset H$, $|C| \leqslant \omega$. Then there exists a sequence $\alpha_{0}, \alpha_{1}, \ldots$ of distinct ordinals such that $C \subset \cup_{n \in \omega} H_{\alpha_{n}}$. For each $n$, let $U_{n} \subset U_{d}^{\prime}$ be an open set in $X$ containing $X_{d(n)}$ such that $e_{\alpha_{k} n} \notin U_{n}$ if $k \leqslant n$. Note $U_{n} \cap \bar{U}_{d}=\varnothing$ if $d \neq d(n)$. Let $V_{n}$ be an open set in $X$ 
containing $X_{d\left(\lambda_{\alpha_{n}}\right)}$ such that $c_{\lambda_{\alpha_{n} k}} \notin V_{n}$ if $k \leqslant n$, and $V_{n} \cap \bar{U}_{d}=\varnothing$ if $d \neq d\left(\lambda_{\alpha_{n}}\right)$.

If $x \in f^{-1}(y)-\cup_{n \in \omega}\left(U_{n} \cup^{\alpha_{n} k} V_{n}\right)$, then there is an open set $O_{x}$ containing $x$ such that $O_{x} \cap \bar{U}_{d}=\varnothing$ if $d \in\{d(n): n \in \omega\} \cup\left\{d\left(\lambda_{\alpha_{n}}\right): n \in \omega\right\}$. Let

$$
O=\cup\left\{O_{x}: x \in f^{-1}(y)-\cup_{n \in \omega}\left(U_{n} \cup V_{n}\right)\right\} \cup\left(\cup_{n \in \omega}\left(U_{n} \cup V_{n}\right)\right)
$$

There is an open set $W$ containing $y$ such that $f^{-1}(W) \subset O$. Suppose $W^{2} \cap C \neq$ $\varnothing$. Then there exists $m$ such that $W^{2} \cap H_{\alpha_{m}} \neq \varnothing$. Choose $n \in \omega$ such that $\left(e_{\alpha_{m} n}^{*}, c_{\lambda_{\alpha_{m} n}}^{*}\right) \in W^{2} \cap H_{\alpha_{m}}$. Then $\left(e_{\alpha_{m} n}, c_{\lambda_{\alpha_{m} n}}\right) \in O^{2}$. Recall $e_{\alpha_{m}} \in C_{d(n)} \subset \bar{U}_{d(n)}$. Thus $e_{\alpha_{m} n} \in U_{n}$, and so $m>n$. Also recall $c_{\lambda_{\alpha_{m} n}} \in C_{d\left(\lambda_{\alpha_{m}}\right)} \subset \bar{U}_{d\left(\lambda_{\alpha_{m}}\right)}$. Thus $c_{\lambda_{\alpha_{m} n}} \in$ $V_{m}$, which means $n>m$, a contradiction. This proves Claim 4. Hence $Y^{2}$ does not have countable tightness, a contradiction which proves the theorem.

Assuming the continuum hypothesis $(\mathrm{CH})$, we have the following corollary.

Corollary $1.5(\mathrm{CH})$. Suppose $f: X \rightarrow Y$ is closed, with $X$ paracompact. Then each $\partial f^{-1}(y)$ is Lindelöf if either $Y^{2}$ is a $k$-space with $t(Y) \leqslant \omega$, or $t\left(Y^{2}\right) \leqslant \omega$.

Proof. Immediate from Theorem 1.4 and the fact that $\omega_{1}$-compact paracompact spaces are Lindelöf [1].

ReMARK. By the proof below, if $Y^{2}$ is a $k$-space with $t(Y) \leqslant \omega$, then $t\left(Y^{2}\right) \leqslant \omega$. Thus the two conditions are not independent.

Proof. Since $Y^{2}$ is a $k$-space, it has the weak topology with respect to the collection of compact subsets of $Y^{2}$; that is $A \subset Y^{2}$ is closed whenever $A \subset C$ is closed in $C$ for every compact subset of $C$ of $Y^{2}$. Each compact subset $C$ of $Y^{2}$ is contained in $\pi(C)^{2}$, where $\pi$ is the projection from $Y^{2}$ onto $Y$. Then $Y^{2}$ has the weak topology with respect to $\left\{\pi(C)^{2} ; C\right.$ is compact in $\left.Y^{2}\right\}$. Since each $\pi(C)$ is a compact space of countable tightness, by a result of V. I. Malyhin [5, Theorem 4], so is each $\pi(C)^{2}$. Then $t\left(Y^{2}\right) \leqslant \omega$.

We do not know if Corollary 1.5 is true without $\mathrm{CH}$. The problem seems to hinge on strengthening the conclusion of Theorems 1.3 and 1.4 by replacing " $c$-compact" with " $\omega_{1}$-compact". It turns out if we add the condition " $Y$ is sequential" to the hypotheses of these theorems, then we can do it.

Theorem 1.6. Suppose $f: X \rightarrow Y$ is closed with $X$ strongly collectionwise Hausdorff and $Y$ sequential. Then each $\partial f^{-1}(y)$ is $\omega_{1}$-compact if either $Y^{2}$ is a quasi-k-space or $t\left(Y^{2}\right) \leqslant \omega$.

Proof. Suppose $Y^{2}$ is a quasi- $k$-space. Since $Y$ is sequential, by [18, Theorem 2.2] $Y^{2}$ is sequential, hence $t\left(Y^{2}\right) \leqslant \omega$. Thus we can assume that $t\left(Y^{2}\right) \leqslant \omega$. Suppose $\partial f^{-1}(y)$ is not $\omega_{1}$-compact. Then there is a closed discrete set $D \subset \partial f^{-1}(y)$ with $|D|=\omega_{1}$. Let $\left\{U_{d} ; d \in D\right\}$ be a discrete collection of open sets in $X$ with $d \in U_{d}$. Then $f\left(\bar{U}_{d}\right)$ is a closed subset of the sequential space $Y$, and is therefore sequential. Since $y$ is not isolated in $f\left(\bar{U}_{d}\right)$, there exists a sequence $y_{d, n} \rightarrow y$, with $y_{d, n} \in f\left(\bar{U}_{d}\right)-$ $\{y\}$ for each $n \in \omega$. Choose $x_{d, n} \in \bar{U}_{d} \cap f^{-1}\left(y_{d, n}\right)$. As in the proof of Theorem 1.3, we can construct $\left\{d(\alpha) ; \alpha<\omega_{1}\right\} \subset D$ and an infinite set $C_{d(\alpha)} \subset\left\{x_{d(\alpha), n} ; n \in \omega\right\}$ such that $f$ is $1-1$ on $\bigcup_{\alpha<\omega_{1}} C_{d(\alpha)}$. Let $X_{d(\alpha)}=\bar{U}_{d(\alpha)} \cap f^{-1}(y)$. Observe that every open set containing $X_{d(\alpha)}$ contains all but finitely many elements fo $C_{d(\alpha)}$, and that 
$f^{-1}(y) \cup\left(\cup_{\alpha<\omega_{1}} C_{d(\alpha)}\right)$ is closed. Thus $Y$ contains a closed copy of the space obtained by identifying the limit points of $\omega_{1}$ convergent sequences. In [4], this space is denoted by $S_{\omega_{1}}$, and it is proved there that $S_{\omega_{1}}^{2}$ is not a $k$-space.

To complete the proof, it is sufficient to show that $S_{\omega_{1}}^{2}$ does not have countable tightness. For each $\alpha<\omega_{1}$, let $S_{\alpha} \subset S_{\omega_{1}}$ be the union of the first $\alpha$ sequences (with limit point). The closure of a countable subset of $S_{\omega_{1}}^{2}$ is contained in some $S_{\alpha}^{2}$. Thus if $S_{\omega_{1}}^{2}$ had countable tightness, then it would have the weak topology with respect to $\left\{S_{\alpha}^{2} ; \alpha<\omega_{1}\right\}$. But each $S_{\alpha}^{2}$ is a $k$-space (cf. $\left.[9,(7.5)]\right)$, so then $S_{\omega_{1}}^{2}$ would be a $k$-space, contradiction.

Corollary 1.7. Suppose $f: X \rightarrow Y$ is closed with $X$ paracompact and $Y$ sequential. Then each $\partial f^{-1}(y)$ is Lindelöf if either $Y^{2}$ is a $k$-space or $t\left(Y^{2}\right) \leqslant \omega$.

The following example shows that the assumption " $Y^{2}$ is a $k$-space" is not sufficient to obtain " $\partial \mathrm{f}^{-1}(y)$ Lindelöf" in Corollary 1.5.

EXAMPLE 1.8. There exists $f: X \rightarrow Y$ closed with $X$ locally compact and paracompact, such that $Y^{2}$ is a $k$-space, but $\partial f^{-1}(y)$ is not Lindelöf for some $y \in Y$.

Proof. For each $\alpha<\omega_{1}$, let $S(\alpha)$ be a copy of ordinal space $\omega_{1}+1$. Let $X$ be the free union of $\left\{S(\alpha): \alpha<\omega_{1}\right\}$. Let $Y$ be the space obtained from $X$ by identifying the point $\omega_{1}$ in each copy to a single point $\infty$. Let $f: X \rightarrow Y$ be the quotient map. Then $X$ is paracompact and locally compact, $f$ is closed, and $\partial f^{-1}(\infty)$ is not Lindelöf. $X$ is a $k$-space (being locally compact), hence so is $Y$.

It remains to prove that $Y^{2}$ is a $k$-space. First we introduce some notation. For each $\alpha, \beta \leqslant \omega_{1}$, let $\beta(\alpha)$ be the image under $f$ of the element of $S(\alpha)$ corresponding to the ordinal number $\beta$. If $\beta<\beta^{\prime} \leqslant \omega_{1}$, let $\left[\beta(\alpha), \beta^{\prime}(\alpha)\right]=\left\{\gamma(\alpha): \beta \leqslant \gamma \leqslant \beta^{\prime}\right\}$, and let $[\beta(\alpha), \infty]=\left[\beta(\alpha), \omega_{1}(\alpha)\right]$.

Suppose $A \subset Y^{2}$, with $A k$-closed, but not closed. Since for each $\alpha, \beta<\omega_{1}$, $[O(\alpha), \beta(\alpha)] \times Y$ and $Y \times[O(\alpha), \beta(\alpha)]$ are clopen $k$-subspaces of $Y^{2}$, it must be true that $(\infty, \infty) \in \bar{A}-A$.

Since $[f(S(O))]^{2} \cap A$ is closed, there exists $\gamma_{0}<\omega_{1}$ such that $\left[\gamma_{0}(O), \infty\right]^{2} \cap A=$ $\varnothing$. Now suppose $\gamma_{\alpha}$ has been defined for all $\alpha<\beta$, where $\beta<\omega_{1}$, in such a way that the following property $P_{\alpha}$ holds.

$$
P_{\alpha}:\left(\beta_{1}\left(\alpha_{1}\right), \beta_{2}\left(\alpha_{2}\right)\right) \in A \quad \text { and } \quad \alpha_{1}, \alpha_{2} \leqslant \alpha \text { implies } \beta_{1}<\gamma_{\alpha_{1}} \text { or } \beta_{2}<\gamma_{\alpha_{2}} .
$$

It is easy to check that $P_{0}$ holds from the way $\gamma_{0}$ has been defined. We will show how to define $\gamma_{\beta}$ in such a way that $P_{\beta}$ holds.

For each $\alpha \leqslant \beta, f(S(\alpha) \times S(\beta)) \cap A$ and $f(S(\beta) \times S(\alpha)) \cap A$ are closed, so there exists $\delta_{\alpha, \beta}<\omega_{1}$ such that

(i) $\gamma_{\alpha} \leqslant \delta_{\alpha, \beta}$;

(ii) $\left(\left[\delta_{\alpha, \beta}(\alpha), \infty\right] \times\left[\delta_{\alpha, \beta}(\beta), \infty\right]\right) \cap A=\varnothing$; and

(iii) $\left(\left[\delta_{\alpha, \beta}(\beta), \infty\right] \times\left[\delta_{\alpha, \beta}(\alpha), \infty\right]\right) \cap A=\varnothing$.

For each $\alpha<\beta$, and each $\beta^{\prime} \in\left[\gamma_{\alpha}, \delta_{\alpha, \beta}\right)$, we have by $P_{\alpha}$ that $\left(\beta^{\prime}(\alpha), \infty\right) \notin A$ and $\left(\infty, \beta^{\prime}(\alpha)\right) \notin A$ and $\left(\infty, \beta^{\prime}(\alpha)\right) \notin A$. Thus there exists $\delta_{\beta^{\prime}, \beta}^{\alpha}<\omega_{1}$ such that

(a) $\left(\left[\delta_{\beta^{\prime}, \beta}^{\alpha}(\beta), \infty\right] \times\left\{\beta^{\prime}(\alpha)\right\}\right) \cap A=\varnothing$ and

(b) $\left(\left\{\beta^{\prime}(\alpha)\right\} \times\left[\delta_{\beta^{\prime}, \beta}^{\alpha}(\beta), \infty\right]\right) \cap A=\varnothing$. 
Let $\gamma_{\beta}=\sup \left\{\delta_{\alpha, \beta}: \alpha \leqslant \beta\right\}+\sup \left\{\delta_{\beta^{\prime}, \beta}^{\alpha}: \alpha<\beta, \gamma_{\alpha} \leqslant \beta^{\prime}<\delta_{\alpha, \beta}\right\}$. Then $\gamma_{\beta}<\omega_{1}$. To show that $P_{\beta}$ holds, we can suppose $\left(\beta_{1}(\alpha), \beta_{2}(\beta)\right) \in A$, with $\alpha \leqslant \beta$. If $\alpha=\beta$, then either $\beta_{1}<\delta_{\beta, \beta} \leqslant \gamma_{\beta}$ or $\beta_{2}<\delta_{\beta, \beta} \leqslant \gamma_{\beta}$, so $P_{\beta}$ holds. If $\alpha<\beta$, we can suppose $\beta_{2} \geqslant \gamma_{\beta}$. Then it must be true that $\beta_{1}<\delta_{\alpha, \beta}$ (by (ii) above). If $\gamma_{\alpha} \leqslant \beta_{1}<\delta_{\alpha, \beta}$, then since $\gamma_{\beta} \geqslant \delta_{\beta_{1}, \beta}^{\alpha}$, we have $\left(\beta_{1}(\alpha), \beta_{2}(\beta)\right) \in\left(\left\{\beta_{1}(\alpha)\right\} \times\left[\delta_{\beta_{1}, \beta}^{\alpha}(\beta), \infty\right]\right) \cap A$, a contradiction. Thus $\beta_{1}<\gamma_{\alpha}$, so $P_{\beta}$ holds.

Thus we can define $\left\{\gamma_{\alpha}: \alpha<\omega_{1}\right\}$ in such a way that $P_{\alpha}$ holds for each $\alpha<\omega_{1}$. Let $U=\left\{\beta(\alpha): \beta>\gamma_{\alpha}, \alpha<\omega_{1}\right\}$. Then $U$ is an open set in $Y$ containing $\infty$. Since $(\infty, \infty) \in \bar{A}$, there exists $\left(\beta_{1}\left(\alpha_{1}\right) \beta_{2}\left(\alpha_{2}\right)\right) \in U^{2} \cap A$. Since $P_{\alpha_{1}+\alpha_{2}}$ holds, either $\beta_{1}<$ $\gamma_{\alpha_{1}}$ or $\beta_{2}<\gamma_{\alpha_{2}}$. But then either $\beta_{1}\left(\alpha_{1}\right) \notin U$ or $\beta_{2}\left(\alpha_{2}\right) \notin U$, contradiction. Thus $Y^{2}$ is a $k$-space.

2. Applications. As applications of results in $\S 1$, we shall consider the products of $k$-spaces and spaces of countable tightness in more special cases.

Definition $2.1[8,16]$. A collection $\mathcal{P}$ of (not necessarily open) subsets of a space $X$ is a k-network for $X$ if, whenever $C \subset U$ with $C$ compact and $U$ open, then $C \subset \cup \mathscr{F} \subset U$ for some finite subcollection $\mathscr{F}$ of $\mathscr{P}$. An $\aleph$-space is a space with a $\boldsymbol{\sigma}$-locally finite $k$-network, and an $\boldsymbol{\aleph}_{0}$-space is a space with a countable $k$-network.

Note that metrizable spaces are $\boldsymbol{\aleph}$-spaces, and separable metrizable spaces are $\boldsymbol{N}_{0}$-spaces.

We say that $X$ is a locally $\boldsymbol{\aleph}_{0}$-space if each point of $X$ has a neighborhood which is an $\boldsymbol{\aleph}_{0}$-space.

THEOREM $2.2(\mathrm{CH})$ Let $f: X \rightarrow Y$ be a closed map. Let $X$ be a paracompact, locally $\boldsymbol{\aleph}_{0}$-space. Then the following are equivalent.

(a) $t\left(Y^{2}\right) \leqslant \omega$

(b) each $\partial f^{-1}(y)$ is Lindelöf;

(c) $Y$ is a locally $\boldsymbol{\aleph}_{0}$-space; and

(d) $Y$ is locally separable.

Furthermore, if $Y$ is sequential, then the $\mathrm{CH}$ assumption can be omitted.

Proof. (a) $\Rightarrow(b)$ : This is Corollary 1.5 .

(b) $\Rightarrow$ (c): Since each subset of a locally $\boldsymbol{\aleph}_{0}$-space is locally $\boldsymbol{\aleph}_{0}$, as in the proof of [7, Corollary 1.2], we can assume that each $f^{-1}(y)$ is Lindelöf. Thus, $f$ is a closed map with each $f^{-1}(y)$ Lindelöf. Then, for each $y \in Y$, there is a closed neighborhood $W$ of $y$ in $Y$, and open subsets $V_{i}$ of $X$ which are $\boldsymbol{\aleph}_{0}$-spaces such that $f^{-1}(W) \subset \cup_{i=1}^{\infty} V_{i}$. Since $\cup_{i=1}^{\infty} V_{i}$ is an $\boldsymbol{\aleph}_{0}$-space, so is $f^{-1}(W)$. Since the closed image of an $\boldsymbol{\aleph}_{0}$-space is also $\aleph_{0}$ by $[8, \mathrm{G}], W$ is an $\aleph_{0}$-space. This implies (c).

(c) $\Rightarrow$ (a) and (c) $\Rightarrow$ (d): By $[8, F], Y^{2}$ is a locally $\boldsymbol{\aleph}_{0}$-space. Then, by $[8, \mathrm{D}, \mathrm{E}] Y^{2}$ is locally a hereditarily separable space. Hence $t\left(Y^{2}\right) \leqslant \omega$.

$(d) \Rightarrow(b)$ : This follows from [21, Proposition 1], because $Y$ is paracompact, hence is locally Lindelöf by (d).

From Theorem 2.2 and some results in [21], we have

COROLlary 2.3. Let $f: X \rightarrow Y$ be a closed map with $X$ locally separable metric. Then the following are equivalent. 
(a) $t\left(Y^{2}\right) \leqslant \omega$

(b) each $\partial f^{-1}(y)$ is Lindelöf;

(c) $Y$ is locally separable;

(d) $Y$ is locally Lindelö;

(e) $Y$ is an $\mathrm{r}$-space.

Definition 2.4. A decreasing sequence $\left(A_{n}\right)$ in a space $X$ is a $k$-sequence [10], if it is an outer network at a compact subset $K$ of $X$; that is, $K=\cap_{n=1}^{\infty} A_{n}$ and every neighborhood of $K$ contains some $A_{n}$. By regularity, if a compact subset $K$ has an outer network, it has one in which each $A_{n}$ is closed in $X$.

Let $Y$ be a space. Then $Y$ satisfies condition $K\left(\boldsymbol{\aleph}_{0}\right)$ [20] if, for any $k$-sequence $\left(A_{n}\right)$ in $Y$, some $A_{n}$ is countably compact.

It is shown that [20, Proposition 2.4] a space $Y$ satisfies $K\left(\boldsymbol{\aleph}_{0}\right)$ if and only if each closed subset of $Y$ which is a paracompact $M$-space is locally compact.

Definition 2.5 [10, Lemma 3.E.2]. A space $Y$ is a $b i-k$-space if, whenever a filter base $\mathcal{F}$ accumulates at $y$ in $Y$, then there exists a $k$-sequence $\left(A_{n}\right)$ in $Y$ such that $y$ $\in \overline{F \cap A_{n}}$ for all $n \in N$ and all $F \in \mathscr{F}$.

It is shown that [10, Theorem 3.E.3] $Y$ is a bi-k-space if and only if $Y$ is a bi-quotient image of a paracompact $M$-space $X$. Then by a result of $\mathrm{H}$. Wicke [23], spaces of pointwise countable type [2] are bi- $k$.

Definition 2.6. A space $X$ is a $k_{\omega}$-space [9] (K. Morita [13] called it a space of class $\left.\mathfrak{S}^{\prime}\right)$, if it has the weak topology with respect to a countable covering of compact subsets of $X$.

For a space $Y$ we shall say that $Y$ is a locally $k_{\omega}$-space, if each point of $Y$ has a neighborhood whose closure is a $k_{\omega}$-space.

It is implicit in a result of $\mathbf{J}$. Milnor [12, Lemma 2.1] that the product of two $k_{\omega}$-spaces is $k_{\omega}$. This fact implies the following lemma.

LeMma 2.7. Let $Y$ be a locally $k_{\omega}$-space. Then $Y^{2}$ is a locally $k_{\omega}$-space, hence, a $k$-space.

LEMMA 2.8. Let $f: X \rightarrow Y$ be a closed map with each $\partial f^{-1}(y)$ Lindelöf. If $X$ is bi-k and $Y$ satisfies $K\left(\boldsymbol{\aleph}_{0}\right)$, then $Y$ is a locally $k_{\omega}$-space.

Proof. Since each closed subset of $X$ is a bi- $k$, as in the proof of [7, Corollary 1.2], we can assume that each $f^{-1}(y)$ is Lindelöf. Let $y \in Y$. Then we will prove that each point of $f^{-1}(y)$ has a neighborhood contained in the inverse image of some compact subset of $Y$. To see this, suppose not. Then there is a point $a_{0}$ of $f^{-1}(y)$ such that for every neighborhood $V$ of $a_{0}$ and for every compact subset $K$ of $Y, V \nsubseteq f^{-1}(K)$.

Let $\mathscr{F}=\left\{X-f^{-1}(K) ; K\right.$ is compact in $\left.Y\right\}$. Then $\mathscr{F}$ is a filter base accumulating at the point $a_{0}$. Since $X$ is bi-k, there exists a $k$-sequence $\left(A_{n}\right)$ in $X$ such that $a_{0}$ $\in \overline{F \cap A_{n}}$ for all $n \in \omega$ and all $F \in \mathcal{F}$. Obviously, $\left(f\left(A_{n}\right)\right)$ is a $k$-sequence in $Y$. Thus, by condition $K\left(\boldsymbol{\aleph}_{0}\right)$, some $f\left(A_{n_{0}}\right)$ is compact. Let $K_{0}=f\left(A_{n_{0}}\right)$. Then, $a_{0}$ $\in \overline{\left(X-f^{-1}\left(K_{0}\right)\right) \cap A_{n_{0}}} \subset \overline{\left(X-f^{-1}\left(K_{0}\right)\right) \cap f^{-1}\left(K_{0}\right)}=\varnothing$. This is a contradiction. 
Thus, each point $x$ of $f^{-1}(y)$ has a neighborhood $V_{x}$ which is contained in the inverse image of some compact subset of $Y$. Since $f^{-1}(y)$ is Lindelöf, $\left\{V_{x}: x \in f^{-1}(y)\right\}$ contains a countable subcover $\left\{V_{n}\right\}_{n \in \omega}$ of $f^{-1}(y)$. For each $n$, let $K_{n}$ be a compact subset of $Y$ such that $V_{n} \subset f^{-1}\left(K_{n}\right)$. Since $f$ is closed and $Y$ is regular there exists a neighborhood $W$ of $Y$ such that $f^{-1}(\bar{W}) \subset \cup_{n \in \omega} V_{n}$. Let $F=f^{-1}(\bar{W})$ and $\mathcal{V}=\{F \cap$ $\left.V_{i}: i \in \omega\right\}$. Then, since $\mathcal{T}$ is an open covering of $F, F$ has the weak topology with respect to $\mathcal{T}$. Since $F \cap V_{i} \subseteq F \cap f^{-1}\left(K_{i}\right)$ for each $i \in \omega, F$ has the weak topology with respect to $\left\{F \cap f^{-1}\left(K_{i}\right) ; i \in \omega\right\}$. Since $f \mid F$ is closed, hence quotient, $f(F)=\bar{W}$ has the weak topology with respect to $\left\{\bar{W} \cap K_{i}: i \in \omega\right\}$. Thus $\bar{W}$ is a $k_{\omega}$-space, and so $Y$ is a locally $k_{\omega}$-space.

Lemma 2.9. Let $f: X \rightarrow Y$ be a closed map with $X$ normal and $t(Y) \leqslant \omega$. If $Y^{2}$ is a $k$-space, then either $Y$ satisfies condition $K\left(\boldsymbol{\aleph}_{0}\right)$ or each $\partial f^{-1}(y)$ is countably compact.

PROof. According to [20, Theorem 4.2], if the product of two spaces is quasi- $k$, and one factor is not an inner-one $A$-space in the sense of E. Michael, R. C. Olson and F. Siwiec [11], then the other factor satisfies $K(\alpha)$, where $\alpha$ is its tightness. $Y$ satisfies condition $K\left(\boldsymbol{\aleph}_{0}\right)$, or $Y$ is an inner-one $A$-space. If $Y$ is inner-one $A$, by [10, Theorem 9.9] each $\partial \mathrm{f}^{-1}(y)$ is countably compact.

LemMA 2.10 [10]. Bi-k-spaces are preserved by perfect images and countable products.

By invoking Corollary 1.5, and Lemmas 2.7, 2.8, 2.9 and 2.10, we obtain the following theorem.

THEOREM $2.11(\mathrm{CH})$. Let $f: X \rightarrow Y$ be a closed map with $X$ paracompact bi-k. If $t(Y) \leqslant \omega$, then the following are equivalent. When $Y$ is sequential, the $\mathrm{CH}$ assumption can be omitted.

(a) $Y^{2}$ is a $k$-space.

(b) $Y$ is locally $k_{\omega}$, or each $\partial f^{-1}(y)$ is compact.

(c) $Y$ is locally $k_{\omega}$, or bi-k.

Corollary 2.12. Let $f: X \rightarrow Y$ be a closed map with $X$ or $Y$ sequential. Let $X$ be a paracompact space of pointwise countable type. Then $Y^{2}$ is a sequential space (equivalently, a $k$-space by [18, Theorem 2.2]) if and only if $Y$ is locally $k_{\omega}$ or bi-k.

Before proceeding with the next lemma, we remind the reader that the perfect image of an $\aleph$-space is an $\aleph$-space, but the closed image of a locally compact metric space need not be $\boldsymbol{\aleph}$-space (cf. [21, Theorem 7]).

LEMMA 2.13. Let $f: X \rightarrow Y$ be a closed map with each $\partial f^{-1}(y)$ Lindelöf. If $X$ is an $\boldsymbol{\aleph}$-space, and $Y$ satisfies condition $K\left(\boldsymbol{\aleph}_{0}\right)$, then $Y$ is also an $\boldsymbol{\aleph}$-space.

Proof. Let $\mathscr{P}=\cup_{i=1}^{\infty} \mathscr{P}_{i}$ be a $\sigma$-locally finite $k$-network for $X$ satisfying the following conditions: Each element of $P$ is closed, $\mathscr{P}_{i} \subseteq \mathscr{P}_{i+1}$ and $\mathscr{P}_{i}$ is closed with respect to finite intersections. Let $K$ be an arbitrary compact subset of $Y$. Since each subset of an $\boldsymbol{\aleph}$-space is an $\boldsymbol{\aleph}$-space, as in the proof of [7, Corollary 1.2], we can assume that each $f^{-1}(y)$ is Lindelöf and that there exists a compact subset $C$ of $X$ with $f(C)=K$. 
Let $\mathscr{P}^{\prime}=\{P \in \mathscr{P}: \mathcal{P} \cap C \neq \varnothing\}$, and let $\mathcal{C}$ be the collection of finite unions of elements of $\mathscr{P}^{\prime}$ which contain the compact subset $C$. Then $C$ is a nonempty, countable collection in $X$.

Let $\mathcal{C}=\left\{P_{i}: i \in \omega\right\}$ and $C_{n}=\bigcap_{i=1}^{n} P_{i}$ for each $n$. Then $\left(C_{n}\right)$ is a $k$-sequence for $C$. Since $\left(f\left(C_{n}\right)\right)$ is a $k$-sequence for $K$, by $K\left(\boldsymbol{\aleph}_{0}\right)$ there exists a compact subset $f\left(C_{n_{0}}\right)$ of $Y$. On the other hand, by the ronditions of the collection $\mathcal{P}$, each $C_{n}$ can be expressed as a union of finitely many elements of $\mathscr{P}$. So, the compact subset $f\left(C_{n_{0}}\right)$ containing $K$ can be expressed as a union of finitely many elements of $f(\mathscr{P})$. Let $\mathcal{K}_{i}=\{f(P): P \in \mathscr{P}$ and $f(P)$ is compact in $Y\}$, and let $\mathcal{K}_{i}^{*}$ be the union of all elements of $\mathcal{K}_{i}$. Then, since $f\left(\mathscr{P}_{i}\right) \subset f\left(\mathscr{P}_{i+1}\right)$, by the above, each compact subset of $Y$ is contained in some $\mathscr{K}_{i}^{*}$.

We will now prove that $Y$ is an $\aleph$-space. Each $\mathcal{K}_{i}$ is a hereditarily closure-preserving collection of compact subsets of $Y$, that is, whenever a subset $K^{\prime}$ of $K$ is chosen for each $K \in \mathscr{K}_{i}$, the collection $\left\{K^{\prime}: K \in \mathscr{K}_{i}\right\}$ is closure-preserving. This is because $\mathcal{K}_{i}$ is the image of a locally finite, hence hereditarily closure-preserving, collection under a closed map. Then by a result of Michael [6, Theorem 1], each $\mathcal{K}_{i}^{*}$ is paracompact. Next, to see each $\mathscr{K}_{i}^{*}$ is locally $\aleph_{0}$, let $\mathscr{T}_{i}=\left\{P \in \mathscr{P}_{i}: f(P) \in \mathscr{K}_{i}\right\}$ and let $\mathfrak{T}_{i}^{*}=\cup \mathfrak{T}_{i}$. Then $\mathfrak{\Re}_{i}^{*}$ has the weak topology with respect to the locally finite closed collection $\mathscr{\Re}_{i}$. Also, $f \mid \mathscr{T}_{i}^{*}$ is closed, hence quotient. Thus $\mathcal{K}_{i}^{*}=f\left(\mathscr{T}_{i}^{*}\right)$ has the weak topology with respect to $\mathcal{K}_{i}$. Since $f$ is closed and each $f^{-1}(y)$ is Lindelöf, $\mathscr{K}_{i}$ is locally countable. Hence each $\mathscr{K}_{i}^{*}$ is a locally $k_{\omega}$-space. Since each compact subset of $X$ is an $\boldsymbol{\aleph}_{0}$-space, by $[\mathbf{8}, \mathrm{G}]$ each compact subset of $Y$ is also $\boldsymbol{\aleph}_{0}$ because it is the image of a compact subset of $X$. Then each $\mathscr{K}_{i}^{*}$ is a locally $\boldsymbol{\kappa}_{0}$-space, since each point has a neighborhood which has the weak topology with respect to a countable collection of compact $\boldsymbol{\aleph}_{0}$-spaces (see [8]). So, each $\mathcal{K}_{i}^{*}$ is a paracompact, locally $\boldsymbol{\aleph}_{0}$-space. It follows that each $\mathcal{K}_{i}^{*}$ is also an $\boldsymbol{\aleph}$-space. As is seen, each compact subset of $Y$ is contained in some $\mathcal{K}_{i}^{*}$. Since each $\mathcal{K}_{i}^{*}$ is an $\boldsymbol{\aleph}$-space, it follows that $Y$ is also an $\aleph$-space. This completes the proof of the lemma.

Lemma 2.14 [19, TheORem 3.1]. Let $Y$ be $a k$-and $\boldsymbol{N}$-space. Then $Y^{2}$ is a $k$-and $\boldsymbol{N}$-space if and only if $Y$ is metrizable, or $Y$ has the weak topology with respect to a countable covering of closed and locally compact subsets of $Y$.

Let a $k$-space $Y$ be the closed image of an $\boldsymbol{K}$-space. Since each closed subset of an $\boldsymbol{K}$-space is easily seen to be a $G_{\delta}$-set, each point of $Y$ is a $G_{\delta}$-set. Thus by [10, Theorem 7.3], $Y$ is sequential. Therefore, by Corollary 1.7, and Lemmas 2.13 and 2.14, we have

THEOREM 2.15. Let $f: X \rightarrow Y$ be a closed map with $X$ a paracompact $\aleph$-space. Then $Y^{2}$ is a $k$-space if and only if $Y$ is metrizable, or $Y$ is an $\mathbf{\aleph}$-space having the weak topology with respect to a countable covering of closed and locally compact subsets of $Y$.

Remark. Let $X$ be an $\boldsymbol{\aleph}$-space each of whose countable (resp. uncountable) subset has an accumulation point. Then $X$ is an $\boldsymbol{\aleph}_{0}$-space, and so $X$ is compact (resp. Lindelöf). Thus, by Theorem 1.6, we have the following.

If an $\boldsymbol{K}$-space $X$ is more generally strongly collectionwise Hausdorff, then the statement of Theorem 2.15 is also valid. 


\section{REFERENCES}

1. G. Aquaro, Point-countable open coverings in countably compact spaces, General Topology and Its Relations to Modern Analysis and Alyebra. II, Academia, Prague, 1966, pp. 39-41.

2. A. V. Arhangel'skii, Bicompact sets and the topology of spaces, Trans. Moscow Math. Soc. 13 (1965), $1-65$.

3. R. Engelking, General topology, PWN, Warsaw, 1977.

4. G. Gruenhage, $k$-spaces and products of closed images of metric spaces, Proc. Amer. Math. Soc. 80 (1980), 478-482.

5. V. I. Malyhin, On tightness and suslin number in $\exp X$ and in a product of spaces, Soviet Math. Dokl. 13 (1972), 496-499.

6. E. Michael, Another note on paracompact spaces, Proc. Amer. Math. Soc. 8 (1957), 822-828.

7. _ A note on closed maps and compact sets, Israel J. Math. 2 (1965), 173-176.

8., $\boldsymbol{\aleph}_{0}$-spaces, J. Math. Mech. 15 (1966), 983-1002.

9. , Bi-quotient maps and cartesian products of quotient maps, Ann. Inst. Fourier (Grenoble) 18 (1968), 287-302.

10. __ A quintuple quotient quest, General Topology Appl. 2 (1972), 91-138.

11. E. Michael, R. C. Olson and F. Siwiec, A-spaces and countably bi-quotient maps, Dissertationes Math. 133 (1976), 4-43.

12. J. Milnor, Construction of universal bundles. I, Ann. of Math. 63 (1956), 272-284.

13. K. Morita, On decomposition spaces of locally compact spaces, Proc. Japan Acad. Ser. A Math. Sci. 32 (1956), 544-548.

14. K. Morita and S. Hanai, Closed mappings and metric spaces, Proc. Japan Acad. Ser. A Math. Sci. 32 (1956), 10-14.

15. J. Nagata, Quotient and bi-quotient spaces of $M$-spaces, Proc. Japan Acad. Ser. A Math. Sci. 45 (1969), 25-29.

16. P. O'Meara, On paracompactness in function spaces with the compact-open topology, Proc. Amer. Math. Soc. 29 (1971), 183-189.

17. A. H. Stone, Metrizability of decomposition spaces, Duke Math. J. 17 (1950), 317-327.

18. Y. Tanaka, On quasi-k-spaces, Proc. Japan Acad. Ser. A Math. Sci. 46 (1970), 1974-1979.

19. $A$ characterization for the products of $k$ - and $\boldsymbol{\aleph}_{0}$-spaces and related results, Proc. Amer. Math. Soc. 59 (1976), 149-154.

20. ___ Some necessary conditions for products of $k$-spaces, Bull. Tokyo Gakugei Univ. (4) 30 (1978), $1-16$.

21. __ Closed maps on metric spaces, Topology Appl. 11 (1979), 87-92.

22. I. A. Vainstein, On closed mappings of metric spaces, Dokl. Akad. Nauk SSSR 57 (1947), 319-321. (Russian)

23. H. Wicke, On the Hausdorff open continuous images of Hausdorff paracompact p-spaces, Proc. Amer. Math. Soc. 22 (1969), 136-140.

Department of Mathematics, Auburn University, Auburn, Alabama 36830

Department of Mathematics, Tokyo Gakugei University, Tokyo, JaPan 